\title{
Übersichtsbeiträge
}

\section{Öko-Audit eines Bio-Agrar-Betriebes}

\author{
Winfried Schröder ${ }^{*}$, Reno Furmanek ${ }^{1}$, Guido Bohmann ${ }^{1}$, Karin Berkhoff ${ }^{1}$, Johannes große Beilage ${ }^{2}$ und \\ Katja Baumann ${ }^{1}$ \\ ${ }^{1}$ Institut für Umweltwissenschaften der Hochschule Vechta, Postfach 15 53, D-49364 Vechta \\ ${ }^{2}$ Biohof Bakenhus des Oldenburgisch-Ostfriesischen Wasserverbandes, Bakenhuser Esch 8, D-26197 Großenkneten
}

* Korrespondenzautor (wschroeder@iuw.uni-vechta.de)

\section{DOI: http://dx.doi.org/10.1065/uwsf2002.09.045}

Zusammenfassung. Die Umweltwirkungen eines nach Naturland-Kriterien arbeitenden landwirtschaftlicher Betriebs werden anhand ausgewählter Kriterien der EG-Öko-Audit-Verordnung VO 1836/93/EWG untersucht. Schwerpunkte sind die Methoden und Ergebnisse des Wirkungsaudits (Kap. 2, 3) sowie die daraus abgeleiteten Empfehlungen, das Umweltprogramm (Kap. 4). Einführend werden das Projekt (Kap. 1.1), untersuchungsrelevante Aspekte der Öko-Audit-Verordnung (Kap. 1.2), der untersuchte Betrieb sowie die Arbeitsschwerpunkte dargelegt (Kap. 1.3). Nur eines der 21 Wirkungsauditkriterien, die Dichte mehrreihiger Gehölze, überschreitet den Toleranzbereich des Bewertungssystems. Die Studie zeigt den Bedarf der Verfahrensharmonisierung auf.

Schlagwörter: Allgemeine Bodenabtragsgleichung; Biodiversität; Bodenerosion; Bodenschutz; GIS; Landschaftsdiversität; ÖkoAudit-Verordnung; Tierschutz; Umweltwirkungen

\section{Projektrahmen}

\subsection{Ziel}

Bewertungen sind eine methodisch anspruchsvolle Aufgabe. Denn bewerten bedeutet, dass ein Sachverhalt empirisch quantifiziert und nachvollziehbar mit einem Bewertungsmaßstab verknüpft werden muss (Schröder 1996, 1998a, 1999; Schröder und Daschkeit 1994, 2002). Anlässlich zunehmender Kritik an der landwirtschaftlichen Produktion sollten die Umweltwirkungen eines landwirtschaftlichen Betriebes in Anlehnung an die Öko-Audit-Verordnung 1836/93/EWG erfasst und bewertet werden. Die Untersuchung fand in einem Studienprojekt statt, das der Korrespondenzautor im Wintersemester 2000/2001 und im Sommersemester 2001 mit Studierenden des Diplomstudiengangs Umweltwissenschaften an der Hochschule Vechta durchführte.

\section{2 Öko-Audit-Verordnung}

Die Verordnung 1836/93/EWG ist als Instrument zur Verbesserung des betrieblichen Umweltschutzes gedacht. Doch nicht nur Gewerbe und Industrie, sondern zunehmend auch die landwirtschaftlichen Betriebe müssen sich den Forderungen der Verbraucher nach einer umweltverträglichen Produktion gesunder Erzeugnisse sowie dem wachsenden ökono-

\section{Abstract}

Eco-audit of a Bio-Farm

Environmental impacts of a farm which is managed in accordance with ecological criteria is assessed. To this end the ecoaudit ordinance of the European Union 1836/93/EWG is used as guiding principle. This article focuses on methods and results of the impact assessment (chapter 2,3) and the recommendations derived from that (chapter 4). Before that, objectives of investigation (chapter 1.1), key topics of the assessment ordinance are summarised (chapter 1.2), and finally the farm and the main fields of investigation are described (chapter 1.3). The density of complex hedge rows, as one from 21 assessment criteria, exceeds the limit of tolerance. The investigation underlines the need for further methodological harmonisation.

Keywords: Animal protection; biodiversity; environmental impact assessment; eco-audit ordinance; GIS; landscape diversity; soil erosion; soil protection; Universal Soil Loss Equation

mischen Druck stellen - auch die ökologisch orientierten unter ihnen. Umweltmanagement- bzw. Umwelt-Audit-Systeme könnten helfen, die landwirtschaftliche Produktion gesundheits- und umweltgerechter zu gestalten (Zellmann et al. 1999a, S. 50 f.).

\subsection{Untersuchter Betrieb und Arbeitsschwerpunkte}

Die im Folgenden vorgestellte Untersuchung ist strukturell an einer Pilotstudie zur Anwendung der EG-Öko-Audit-Verordnung auf Schweinemastbetriebe orientiert. Diese umfasst folgende Verfahrensschritte nach VO 1836/93/EWG (Schneider 1999):

- Umweltpolitik: Festlegung unternehmensbezogener Gesamtziele und Handlungsgrundsätze zum Umweltschutz;

- Soll-Anforderungen: Festlegung des Untersuchungsrahmens und Zusammenstellung der relevanten Umweltgesetze und betriebsspezifischer Produktionsvorschriften;

- Ermittlung des Ist-Zustandes: Erfassung der umweltrelevanten betrieblichen Tätigkeit, Erstellung einer Betriebsbilanz, Darstellung der Ergebnisse;

- Soll-Ist-Vergleich: Gegenüberstellung von Betriebswerten und gesetzlichen Rahmenbedingungen, Ursachenanalyse für den Ist-Zustand, Entwicklung eines Schwachstellenprofils;

- Umweltprogramm: Maßnahmenkatalog zum Abbau der Schwachstellen; 
- Umweltmanagementsystem: weitere Verfahrensschritte, die in dem Studienprojekt nicht bearbeitet wurden;

- Umweltbetriebsprüfung;

- Umwelterklärung.

Untersuchungsobjekt des Studienprojekts war der Biohof Bakenhus des Oldenburgisch-Ostfriesischen Wasserverbandes in Großenkneten (Landkreis Oldenburg). Er weist folgende Bewirtschaftungsformen und Betriebszweige auf:

- Kontrolliert ökologischer Landbau (nach Naturland-Verband)

- 70\% Weide / Mähgrünland, 30\% Getreide / Ackerfutter auf insgesamt 175 ha,

- Mutterkuh- und Rinderhaltung auf der Weide und im Tieflaufstall mit Auslauf / Offenfront (180 Tiere),

- Mast-Schweinehaltung auf Stroh mit Auslauf (90 Tiere),

- Bio-Fleischerei mit Hofladen und Ladengeschäft in der Stadt Vechta,

- Pensionspferdehaltung (32 Plätze, z.Zt. 4 Plätze für Auslaufhaltung) sowie

- Reitanlage mit Reithalle.

Arbeitsschwerpunkte des Studienprojekts waren das Wirkungs-, das Management- sowie das Rechtsaudit (Zellmann et al. 1999b, S. 182). Jedoch werden das Management- und das Rechtsaudit hier nicht dargestellt. Alle Daten wurden in dem eigens angelegten ArcView-GIS Biohof Bakenhus verwaltet.

\section{Methoden zur Bewertung von Umweltauswirkungen}

Die Auswirkungen des Biohofs Bakenhus auf die Umwelt werden anhand von Agrar-Umweltindikatoren, Stoff- und Energiebilanzen, Kenngrößen bzw. Maßzahlen sowie Checklisten mit den betrieblichen Informationstechniken analysiert (Gebauer und Bäuerle 2000, S. 452). Für die Bewertung der solcherart quantifizierten Umweltwirkungen stehen neben dem Ansatz von Schneider (1999) weitere Verfahren zur Verfügung: die Modelle KUL (Kritische Umweltbelastungen Landwirtschaft) und REPRO (Gebauer und Bäuerle 2000, S. 462 ff.). REPRO ist ein EDV-gestütztes System für die Analyse, Bewertung und Steuerung von Stoff- und Energieflüssen landwirtschaftlicher Betriebe und berücksichtigt Betriebsstruktur, Bewirtschaftungsintensität und der Verfahrensabläufe. Grundlage der Bewertung sind Agrarumweltindikatoren, denen Optimumbereiche zugeordnet werden (Dubsky et al. 1997, S. 2). REPRO konnte für das Umweltaudit des Biohofs Bakenhus nicht eingesetzt werden, da nicht alle Datenanforderungen des Modells erfüllt werden konnten.

KUL ist ein EDV-gestütztes Verfahren zur Erfassung und Bewertung der Umweltverträglichkeit landwirtschaftlicher Produktionssysteme anhand 20 definierter Kriterien in den Kategorien Nährstoffhaushalt, Bodenschutz, Pflanzenschutzmitteleinsatz, Landschafts- und Artenvielfalt sowie Energiebilanz. Die Bewertung der einzelnen Kriterien erfolgt über Boniturnoten. Das anzustrebende Optimum wird mit der Bonitur 1, eine maximal tolerable Situation mit der Bonitur 6 bewertet. Umweltbelastungen werden als Überschreitungen der Toleranzbereiche definiert. Somit ermöglicht KUL eine Bewertung der Umweltrelevanz in Form von Noten und Toleranzbereichen. Gleichzeitig werden Prioritäten für erforderliche Maßnah- men zum Ausdruck gebracht (Eckert und Breitschuh 1997, S. 185 ff.; Gebauer und Bäuerle 2000, S. 456 f).

KUL ist kein originärer Bestandteil des Öko-Audits gemäß des Environmental Management and Audit Scheme EMAS (ISO 14001), sondern eine eigenständige Methode zur Bewertung umweltverträglicher Landbewirtschaftung mit geringem Implementierungs- und Erfassungsaufwand. Deshalb wurde KUL in einem Öko-Audit zur Erfassung und Bewertung der Umweltwirkungen an sechs landwirtschaftlichen Betrieben erprobt. Dabei erwies sich KUL als geeignetes Instrument, allerdings wurde eine stärkere Berücksichtigung von Grünland- und Tierproduktion sowie die Energiebilanzierung für Ökobetriebe als wünschenswert eingeschätzt (VAFB, Kap. 4 f.). Die sehr stark ackerbauliche Orientierung von KUL schränkt seine Eignung für eine ökologische Gesamtbewertung landwirtschaftlicher Betriebe ein (Schneider 1999, S. 87). Deshalb schlagen Geier und Köpke (2000) die Veränderung von KUL gemäß Tabelle 1 vor. Diese modifizierte KULVersion ist eng an der Umweltverträglichkeit orientiert und schließt Einflüsse durch Produktivitätsanforderungen aus, da ansonsten Betriebe mit extensiver Bewirtschaftung, ertragsschwachen Standorten und mit hohen Leistungen für Landschafts- und Artenvielfalt und ökologisch wirtschaftende Betriebe benachteiligt würden. Für das Öko-Audit des Biohofs Bakenhus wurde die gemäß Tabelle 1 veränderte KUL-Version verwendet. Zusätzlich wurden weitere Merkmale und Bewertungsmethoden verwendet, um ein möglichst umfassendes Bild über die gesamtbetriebliche Situation zu erhalten. Diese Veränderungen werden in Kap. 3.2 erläutert.

\section{Ergebnisse}

Die Umweltwirkungen des Biohofs Bakenhus wurden anhand der in Tabelle 2 aufgeführten Indikatoren festgestellt und durch die Verwendung betrieblicher Bewertungsverfahren eingestuft.

\subsection{Biodiversität}

Die Bewertung der Biodiversität erfolgt in KUL in der Kategorie Landschafts- und Artenvielfalt (siehe Tabelle 1, Zf. 4). Als Kriterien dienen dabei der Flächenanteil ökologischer und landeskultureller Vorrangflächen (ÖLV) sowie die Kulturartendiversität (Eckert et al. 1999, S. 11 f.). Plachter (zit. in BMELF 2000 b, S. 77 f.) kritisiert, dass dieser Ansatz zu reduktionistisch und deshalb kein Fachinstrument des $\mathrm{Na}$ tur- und Umweltschutzes ist. Die Projektgruppe entschied sich daher, beim Umweltwirkungsbereich Biodiversität von der Bewertung mit KUL abzusehen und das Verfahren nach Frieben (1998) anzuwenden. Dieses Bewertungsverfahren hat das Ziel, Defizite, Leistungen und den Optimierungsbedarf organisch wirtschaftender Betriebe im Hinblick auf den Biotop- und Artenschutz und die Stabilität des Agrarökosystems zu ermitteln. Die Grundlage des Bewertungssystems sind Leitbilder, die auch an der ökonomisch nachhaltigen Nutzbarkeit der Ökosysteme orientiert sind. Vor Ort vorgefundener Zustände der Biotope werden mit den in Tabelle 2 (Zf. 1) genannten Kriterien am Leitbild gemessen. Den Er- 
Tab. 1: KUL-Kriterien und Optimierungsvorschläge nach Geier und Köpke (2000)

\begin{tabular}{|c|c|c|}
\hline KUL-Kriterien, verändert nach Geier \& Köpke (2000) & Kommentar zu den Änderungsvorschlägen & Gewichtung (\%) ${ }^{a}$ \\
\hline $\begin{array}{l}1 \text { Nährstoffhaushalt } \\
\mathrm{N}-\text { Saldo (Fläche) } \\
\mathrm{NH}_{3} \text {-Emission (Tiere) } \\
\text { K-Saldo } \\
\text { Gehaltsklasse P } \\
\text { Gehaltsklasse K } \\
\text { Gehaltsklasse Mg } \\
\text { Boden pH-Stufe } \\
\text { Humussaldo }\end{array}$ & $\begin{array}{l}\text { Kriterium streichen } \\
\text { Kriterium streichen } \\
\text { Kriterium streichen }\end{array}$ & $24(28)$ \\
\hline $\begin{array}{l}2 \text { Bodenschutz } \\
\text { Erosionsdisposition } \\
\text { Verdichtungsgefährdung } \\
\text { Median Feldgröße }\end{array}$ & & $15(18)$ \\
\hline $\begin{array}{l}3 \text { Pflanzenschutz } \\
\text { Integrierter Pflanzenschutz } \\
\text { PS-Intensität }\end{array}$ & $\begin{array}{l}\text { Nur Betriebe mit PSM-Einsatz } \\
\text { Bewertung mit toxikol. Methode }\end{array}$ & $10(12)$ \\
\hline $\begin{array}{l}4 \text { Landschafts- und Artenvielfalt } \\
\text { Anteil ÖLV } \\
\text { Kulturartendiversität } \\
\text { Intensität der Ackernutzung } \\
\text { Intensität der Grünlandnutzung } \\
\text { Qualität der ÖLV-Flächen }\end{array}$ & $\begin{array}{l}\text { Bezogen auf die Betriebsfläche } \\
\text { Neu, Summenindikator }^{\text {b) }} \\
\text { Neu, Summenindikator }^{\text {b) }} \\
\text { Neu, Summenindikator }^{\text {b) }}\end{array}$ & $24(28)$ \\
\hline $\begin{array}{l}5 \text { Energiebilanz } \\
\text { Gesamtbetrieb Energieinput } \\
\text { Gesamtbetrieb Energiesaldo } \\
\text { Pflanzenbau Energieinput } \\
\text { Pflanzenbau Energiesaldo } \\
\text { Pflanzenbau Energieeffizienz } \\
\text { Tierhaltung Energieinput } \\
\text { Tierhaltung Energiesaldo } \\
\text { Tierhaltung Energieeffizienz }\end{array}$ & $\begin{array}{l}\text { Kriterium streichen } \\
\text { Kriterium streichen } \\
\text { Neu, differenziert nach Verfahren } \\
\text { Kriterium streichen } \\
\text { Neu, differenziert nach Verfahren }\end{array}$ & $7(8)$ \\
\hline $\begin{array}{l}6 \text { Kulturpflanzen- / Nutztier-Diversität } \\
\text { Nutzung gefährdeter Nutztierrassen } \\
\text { Nutzung gefährdeter Kulturpflanzenarten oder -sorten }\end{array}$ & Neue Kategorie & $5(6)$ \\
\hline $\begin{array}{l}7 \text { Tiergerechtheit (Tierschutz) } \\
\text { Tiergerechtheitsindex }\end{array}$ & $\begin{array}{l}\text { Neue Kategorie } \\
\text { Messung mit dem TGI } 200\end{array}$ & $15(0)$ \\
\hline
\end{tabular}

Tab. 2: Messgrößen und Umweltwirkungsbereiche

\begin{tabular}{|c|c|}
\hline Umweltwirkungsbereich & Messgröße \\
\hline 1 Biodiversität (Kap. 3.1) & $\begin{array}{l}\text { - Verhältnis zwischen Acker- und Dauergrünland } \\
\text { - Düngungsniveau } \\
\text { - Fruchtfolge } \\
\text { - Gesamtanteil theoretisch nicht besiedelbarer Ackerinnenfläche } \\
\text { - Verteilungsdichte mehrreihiger Gehölze / Hecken (lfdm / ha LF) } \\
\text { - Vorhandensein von Sonderbiotopen, Anteil bedeutsamer Landschaftsbestandteile } \\
\text { (Frieben 1998), zusätzlich: } \\
\text { - Art und Zusammensetzung des Viehbestands }\end{array}$ \\
\hline 2 Bodenschutz (Kap. 3.2) & $\begin{array}{l}\text { - Erosion } \\
\text { - Bodenverdichtung } \\
\text { - Schlaggröße }\end{array}$ \\
\hline 3 Emissionen (Kap. 3.3) & - Emittiertes Ammoniak $\left(\mathrm{NH}_{3}\right)$ \\
\hline 4 Nährstoffhaushalt (Kap. 3.3, 3.4) & $\begin{array}{l}\text { Gesamtbilanzen (= Hoftorbilanzen) z.B. für Stickstoff anhand } \\
\text { - Futtermittelzukauf } \\
\text { - Strohzukauf } \\
\text { - Tierzukauf } \\
\text { - Zukauf von Saatgut } \\
\text { - Legume Stickstoffbindungen } \\
\text { - Atmosphärische Stickstoffeinträge } \\
\text { - Verkauf von Tieren und tierischen Produkte } \\
\text { - Verkauf pflanzlicher Produkte } \\
\text { - Selbsterzeugte Futtermittel } \\
\text { - Humusbilanz }\end{array}$ \\
\hline 5 Tierhaltung (Kap. 3.5) & - Tiergerechtheitsindex TGI 200 (Sundrum et al. 1994) \\
\hline
\end{tabular}


Tab. 3: Bewertung des Umweltwirkungsbereiches 'Biodiversität' auf dem Biohof Bakenhus

\begin{tabular}{l|c|c}
\multicolumn{1}{c|}{ Kriterien } & Erreichbarer Höchstwert & Erreichte Punktzahl \\
\hline Verhältnis Acker- zu Dauergrünland [Ackerflächenanteil (\%)] & 5 & 2 \\
\hline Düngungsniveau (kg N/ha) (80 kg N/ha =1 DE/ha) & 5 & 5 \\
\hline Fruchtfolge & 8 & 4 \\
\hline Gesamte theoretisch nicht besiedelbare Ackerinnenfläche (\%) & 5 & 3 \\
\hline Dichte mehrreihiger Gehölze (m/ha) & 5 & 0 \\
\hline Präsenz von Sonderbiotopen (max. 5 Punkte) & 5 & 3 \\
\hline Summe Biohof Bakenhus & & 17 \\
\hline
\end{tabular}

füllungsgraden dieser Kriterien wird über definierte $\mathbf{Z u}$ stands-Wertigkeits-Relationen eine Wertschätzung zugeordnet, die auf Basis von Referenzdaten und umfangreicher $\mathrm{Li}$ teraturauswertung festgelegt wurde (Plachter, in: BMELF 2000 b, S. 77 f.).

Die Übertragung der Punktbewertung nach Frieben (1998) in die Boniturnoten von KUL wurde nach folgendem Schema vorgenommen: Geht man davon aus, dass keiner der von Frieben (1998) untersuchten Betriebe 50\% der Optimalpunktzahl erreicht hat, kann man den Wertebereich von $100 \%$ bis $50 \%$ der erreichbaren Punktzahl der KULBoniturnote 1 zuordnen. Frieben (1998) macht keine Angaben zu Toleranzbereichen für die einzelnen Kriterien, so dass von einem Toleranzbereich von $50 \%$ bis $25 \%$ der Optimalpunktzahl ausgegangen wird. Auf diesen Toleranzbereich verteilen sich die Boniturnoten 2 bis 6 in gleichem Abstand. Werte unterhalb der $25 \%$-Schwelle werden als 'nicht tolerierbar' mit einer Punktzahl von 7 bis 10 dargestellt (siehe Tabelle 5). Der Biohof Bakenhus erreicht bei dem Bewertungskriterium Biodiversität 17 Punkte (Tabelle 3). Dieses Ergebnis ist im Vergleich zu anderen Betrieben als gut einzustufen(Frieben 1998, S. 180 ff.). Optimierungsbedarf herrscht aber vor allem bei der Dichte mehrreihiger Gehölze und beim Verhältnis Acker- zu Dauergrünland.

\subsection{Bodenschutz}

Das KUL-Konzept sieht für die Erfassung und Bewertung der Kategorie Bodenschutz die drei Kriterien Erosion (Kap. 3.2.1), Bodenverdichtung (Kap. 3.2.2) und Schlaggröße (Kap. 3.2.3) vor.

\subsubsection{Erosion}

Die Erosionsgefährdung wird mit der aus den USA übernommenen Allgemeinen Bodenabtragsgleichung (ABAG) ermittelt (Auerswald und Schwertmann 1988, Wishmeier und Smith 1978). Entscheidend für Bodenabtrag ist das Verhältnis von der kinetischen Energie des Transportmediums Wind oder Wasser (Scherkraft; hier: R) und dem Zusammenhalt (Scherfestigkeit) des überströmten Materials (hier: $\mathrm{K})$. Wenn $\mathrm{R}>\mathrm{K}$, dann wird Boden in Abhängigkeit der von Hjulström (1935) für den Wassertransport modellhaft quantifizierten Ausprägungen zweier wesentlicher Steuergrößen von $\mathrm{R}$ und $\mathrm{K}$, nämlich Fließgeschwindigkeit und Textur, losgelöst, transportiert und andernorts abgelagert. Die ABAGFaktoren $\mathrm{L}, \mathrm{S}$ und $\mathrm{C}$ sind weitere Randbedingungen, die $\mathrm{R}$ und $\mathrm{K}$ beeinflussen und deshalb sinnvollerweise zu berücksichtigen sind (Schröder 1998b, S. 114 f.; White et al. 1984).

$A=R \times K \times L \times S \times C \times P$

mit: A Abtrag [t/ha/a ]

$R$ Regenerosivität [N/h/a ]

K Bodenerodierbarkeit [ $(\mathrm{t} / \mathrm{ha}) \times(\mathrm{h} / \mathrm{N})]$

$L$ Hanglängenfaktor (dimensionslos)

S Hangneigungsfaktor (dimensionslos)

C Bewirtschaftungs- und Bedeckungsfaktor (dimensionslos)

P Schutzfaktor erosionsmindemder Maßnahmen (dimensionsios)

Die verwendeten Klimadaten stammen aus folgenden Quellen:

- Institut für Umweltwissenschaften (IUW) der Hochschule Vechta,

- Daten des Deutschen Wetterdienstes (DWD) für Großenkneten, Oldenburg, Diepholz und Osnabrück (monatliche Mittel der Jahre 1961-1990),

- Institut für Strukturforschung und Planung in agrarischen Intensivgebieten (ISPA) der Hochschule Vechta,

- Messdaten der Klimastation des ISPA (10-minütige Messungen im Zeitraum Juli 1994-2000),

- Niedersächsisches Landesamt für Ökologie sowie

- Luftgüte-Überwachungsnetz (30-Minuten-Messungen der Station Cloppenburg 1999-2000).

\subsubsection{Regenerosivität (R)}

Der $R$-Faktor eines Jahres für einen Standort ergibt sich aus der Summation aller erosiven Niederschläge eines Jahres (http://mapserver.ilpoe.uni-stuttgart.de:81/krabag/abag.html). Die Berechnung erfolgte durch Gl. 2 (Auerswald und Schwertmann 1988, S. 4) anhand der von 1961 bis 1990 gemittelten Niederschläge von Mai bis Oktober (Station Großenkneten des Deutschen Wetterdienstes).

$R=0,14 \times N_{S}$

(GI. 2)

mit: $\quad \mathrm{N}_{\mathrm{S}}$ stündliches Niederschlagsmittel im Sommer [mm $\left./ \mathrm{h}\right]$, hier ersetzt durch:

$\mathrm{N}_{\mathrm{S}}$ Summe der Monatsmittel der Sommerniederschläge, um einen R-Faktor für das gesamte Sommerhalbjahr zu erhalten).

Nach Gl. 2 ergibt sich für den $R$-Faktor Sommerniederschlag $_{\text {ein }}$ Wert von $R=0,14 \times 374,6=52,444$. Dieser Wert liegt innerhalb des vorgegebenen Rahmens gemäß der Bodenkundlichen Kartieranleitung (BGR 1982, Tabelle 62).

\subsubsection{Bodenerodierbarkeit (K)}

Die Bodenerodierbarkeit steht in unmittelbarem Zusammenhang mit der Bodenzusammensetzung und -permeabilität und wird durch Gl. 3 ausgedrückt (Schwertmann et al. 1987, S. 19). 
$\mathrm{K}=2,77 \times 10-6 \times \mathrm{M} 1,14 \times(12-\mathrm{OS})+0,043 \times(\mathrm{A}-2)+0,033 \times(4-\mathrm{D}) \quad$ (GI. 3a) mit: $M \quad(\%$ Schluff $+\%$ Feinstsand $) \times(100 \%$ Ton $)$

OS $\%$ organische Substanz (falls OS $>4$, dann setze OS $=4$ )

A Aggregatklasse gem. Bodenkundlicher Kartieranleitung

D Permeabilitätsklasse gem. Bodenkundlicher Kartieranleitung

Eine genauere Abschätzung des Feinstsand-Anteils liefert Formel 3b (BGR 1982, S. 301; Schwertmann et al. 1987, S. 19).

$$
\begin{gathered}
\% \text { Feinstsand }=2,6+0,285 \times(\% \text { Feinsand })-0,138 \\
\times(\% \text { Mittelsand })
\end{gathered}
$$

Die Flächen des Biohofs Bakenhus liegen größtenteils im Bereich trockener, nährstoffarmer und verwehbarer Sandböden, die als Podsol-Braunerden und Podsole ausgeprägt sind. Nachrangig treten Braunerden bzw. Pseudogley-Braunerden auf mäßig trockenen bis frischen Standorten auf (LWK WE 1999, Biohof Bakenhus 2000). Zur Quantifizierung der Variable $M$ in Gl. 3a wurden Bodenanalysen einer Referenzfläche des Biohofes Bakenhus verwendet (FOSVWE 2000). Die Variablen $O S, A$ und $D$ (Gl. 3a) wurden aus den Bodenarten in Verbindung mit Richtwerten aus der Bodenkundlichen Kartieranleitung berechnet. Die abschließende Kalkulation der Bodenerodierbarkeit erfolgte in dem ArcView GIS Biohof Bakenhus und ergab Werte zwischen 0 und 0,328. Diese Ergebnisse entsprechend denen anderer Untersuchungen sandiger bis léhmig-sandiger Braunerden (Schwertmann et al. 1987, S. 24; BGR 1982, Tabelle 95).

\subsubsection{Hanglänge /-neigung (L, S)}

Der zu erwartende Bodenverlust eines Wassererosionsereignisses ist unmittelbar abhängig von der Hanglänge und der Hangneigung. Je stärker das Gefälle und je länger der Hang ist, desto mehr Abtrag ist zu erwarten. Entsprechend wird die Wirkung dieser beiden Faktoren in der ABAG durch eine gemeinsame Relation berücksichtigt:

$$
\begin{aligned}
\mathrm{L} \times \mathrm{S}= & (\mathrm{I} / 22)^{\mathrm{m}} \times\left(65,41 \times \sin ^{2}(\mathrm{H})+4,56 \times \sin (\mathrm{H})+0,065\right) \quad(\mathrm{Gl} .4) \\
\text { mit: } \quad & \mathrm{I} \text { Hanglänge }[\mathrm{m}] \\
& \mathrm{m} \text { Hanglängenexponent (variiert mit der Hangneigung) } \\
& \mathrm{H} \text { Hangneigung }\left[^{\circ}\right]
\end{aligned}
$$

Anhand eines Nomogrammes zur Ermittlung des LS-Faktors in Abhängigkeit von der Hangneigung und der Hanglänge lassen sich die Werte für $m$ per Rückrechnung aus der Hangneigung ermitteln (Schwertmann et al. 1987, S. 31). Die Hanglänge wurde mit dem im Projekt entwickelten GIS Biohof Bakenhus aus der geradlinigen Verbindung zwischen dem höchsten und dem niedrigsten Punkt des Schlages ermittelt (bzw. mehrerer zusammenhängender Schläge, die in diesem Falle gemeinsam betrachtet werden). Die Berechnung der Neigung erfolgte dann anhand der Steigung entlang dieser Linie. Die Ergebnisse für den LS-Faktor liegen zwischen 0,159 und 0,4 .

\subsubsection{Bewirtschaftungs- und Bedeckungsfaktor $\mathrm{C}$}

Der C-Faktor drückt den Einfluss der Bedeckung durch Kulturpflanzen und den der Bodenbearbeitung auf die Erosionsgefährdung aus. Für die Ermittlung der Fruchtfolge eines Schlages werden über drei Jahre hinweg die jeweiligen C-Faktoren der einzelnen Perioden ermittelt, summiert und durch die Zahl der betrachteten Jahre geteilt. Somit erhält man einen durchschnittlichen jährlichen C-Faktor, der in die ABAG eingeht. Für die Ermittlung der Fruchtfolgen auf den einzelnen Schlägen des Biohofes wurden die Daten des Maßnahmenkatalogs der Bodenbearbeitung (Zeitpunkt der Aussaat bzw. Ernte, Art der Bodenbearbeitung) aus der Ackerschlagkartei 1998 bis 2000 ausgewertet. Sind Flächen nicht im gesamten Zeitraum dieser drei Jahre genutzt worden, so wurden nur die vorhandenen Nutzungszeiten berücksichtigt. Die Ergebnisse für den Bewirtschaftungs- und Bedeckungsfaktor $C$ der einzelnen Schläge des Biohofes liegen zwischen 0,03 und 0,4 .

\subsubsection{Schutzfaktor erosionsmindernder Maßnahmen}

Der P-Faktor stellt ein Maß für erosionsmindernde Feldbearbeitung wie Bearbeitungsrichtung oder Terrassierungsmassnahmen dar, verknüpft mit Hanglänge und -neigung (Schwertmann et al. 1987, S. 54). Darüber hinaus können auch besondere Pflanzungsmaßnahmen wie z.B. die Streifensaat in die Ermittlung des P-Faktors einfließen. Auf dem Biohof Bakenhus werden keine bearbeitungstechnischen Erosionsminderungsmaßnahmen durchgeführt, so dass der PFaktor mit einem Wert von 1 in die Berechnung eingeht.

\subsubsection{Nach ABAG berechnete Erosionsgefährdung}

Nach Multiplikation aller von der ABAG vorgegebenen Werte ergeben sich für die Schläge des Biohofes prognostizierte Bodenabträge von 0 bis 2,5 t/ha/a (Abb. 1). Der Mittelwert der berechneten Abtragsraten liegt bei 0,4094 t/ha/a. Damit fällt dieser Wert für den Biohof Bakenhus unter die geforderten 2-10 t / ha pro Jahr (Eckert und Breitschuh 1997, S. 186). Der Aufbau der Boniturnoten-Skala gemäß KUL orientiert sich an der erosiven Grundgefährdung des Biohofes $(0,083 \mathrm{t} / \mathrm{ha} / \mathrm{a})$ und ergibt eine Boniturnote von 2 für den Bereich Erosion.

Die ABAG dient zur Schätzung der Abtragsraten durch Wassererosion, berücksichtigt aber nicht die Winderosion. Somit ist die Bewertung der Erosionsgefährdung unvollständig. Aufgrund der geomorphologischen und klimatischen

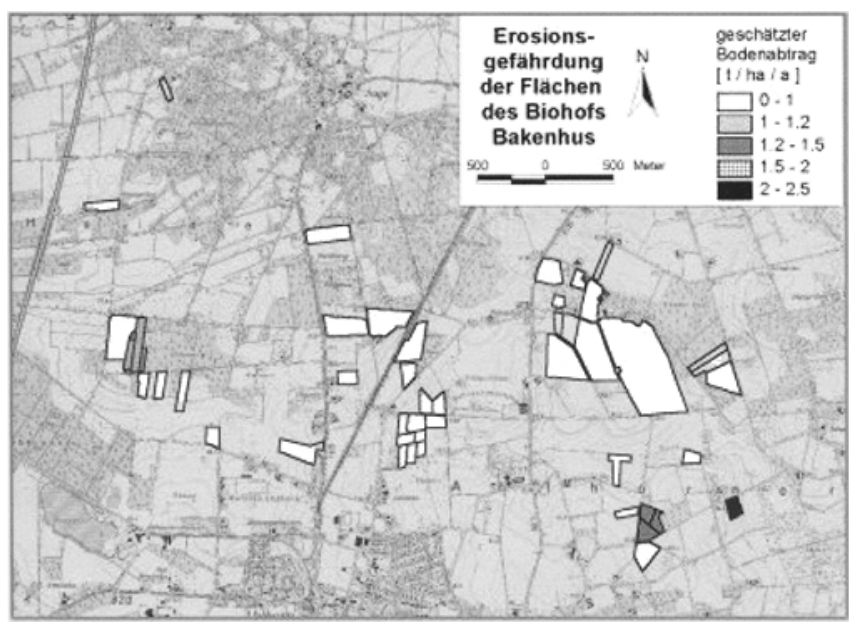

Abb. 1: Prognostizierte Bodenabträge für die Schläge des Biohofes Bakenhus (eigene Darstellung) 
Merkmale der untersuchten Flächen kommt der Winderosion eine größere Bedeutung als der Wassererosion zu. Laut Angaben der Landwirtschaftskammer Weser-Ems sind im Landkreis Oldenburg $0,5 \%$ der landwirtschaftlich genutzten Flächen von Wassererosion betroffen, wesentlich mehr (4,4\%) von Winderosion (LK OL, S. 146 ff.; BMELF 1991, S. 38). Sobald wissenschaftlich geprüfte, praktikable Berechnungsmethoden zur Ermittlung der Winderosion vorliegen, sollte diese in die Erosionsberechnung eingehen.

\subsubsection{Bodenverdichtung}

Es lagen lediglich für einen Schlag, die bereits erwähnte Testfläche des FOSVWE) Daten für die von KUL vorgesehene Berechnung der Bodenverdichtung nach Paul (1999) vor (Korngrößenzusammensetzung, Horizontierung des Bodens, Porenvolumen und die Nutzbare Feldkapazität bei differenten Feuchtegraden). Folglich wurde nur für diese Parzelle eine Verdichtungsgefährdung ermittelt. Die Abschätzung ergab, dass eine Bearbeitung der Testfläche bei feuchtem Boden lediglich mit leichten Maschinen durchgeführt werden darf. Mit zunehmender Trockenheit des Bodens steigt hingegen die Belastbarkeit und damit die duldbare Belastung durch die Auflast des Maschinengewichts.

\subsubsection{Schlaggröße}

Als Kenngröße für die Bewertung der Schlaggröße wird gemäß KUL der Median angesetzt. Dieser teilt die Gesamtzahl aller Schläge ihrer Größe nach in zwei gleich große Teilmengen auf und ist somit ein Maß für die überwiegende Parzellengröße. Als Optimum für diesen Wert wird eine Flächengröße von bis zu 10 ha angesehen (Eckert und Breitschuh 1997, S. 186). Im Toleranzbereich liegen noch Werte, die kleiner als 40 ha sind, wobei jedoch auch Grenzwerte von bis zu 30 ha in der Literatur zu finden sind (Geier und Köpke, in: BMELF 2000a, S. 71). Die Ermittlung des Medians der Schlaggrößen erfolgte mit Microsoft Excel. Datengrundlage sind die Flächendaten aus der Ackerschlagkartei 2000 sowie die im ArcView-GIS Biohof Bakenhus ArcView ermittelten Größen der digitalisierten Schläge. Der Median schwankt je nach Berücksichtigung der relativen Lage der Schläge zueinander zwischen einem Bereich von 2.1 bis 3.4 ha und liegt damit deutlich unter der Obergrenze des nach Eckert und Breitschuh (1997, S. 186) optimalen Bereichs von bis zu 10 ha. Dem Biohof Bakenhus wird somit für die Schlaggröße die Boniturnote 1 zugewiesen.

\subsection{Nährstoffhaushalt und Emissionen}

Eine Expertenbefragung (Münchhausen und Nieberg 1994) ergab, dass für die Beurteilung des Nährstoffhaushaltes sowohl einzelbetrieblich als auch für eine Region der durch eine Flächenbilanz ermittelte Stickstoffsaldo als geeigneter AgrarUmweltindikator erachtet wird. Doluschitz et al. (1992) sehen in der Stickstoffbilanzierung landwirtschaftlicher Betriebe die Möglichkeit des Einstiegs in eine ökologische Buchführung. Mit Nährstoffbilanzen können Stoffkreisläufe nachvollzogen und potentielle Verluste sowohl kleinräumig für einzelne Flächen und Betriebe als auch für große Agrarräume quantitativ ermittelt werden (Hülsbergen und Biermann 1994).

Die Problembereiche Eutrophierung und landwirtschaftliche Emissionen werden bei KUL in der Kategorie Nährstoffhaushalt anhand der Kriterien N-, P-, K-Saldo, $\mathrm{NH}_{3}$-Emissionen, Gehaltsklassen der Nährstoffe P, K, Mg, Boden-pHStufe und Humussaldo betrachtet (siehe Tabelle 2, Zf. 1). Zusammenfassend stellt Abb. 2 die KUL-Verfahrensweise zur Stickstoff-Bilanzierung dar. In Tabelle 4 werden die ermittelten Betriebswerte des Biohofes Bakenhus in der KUL-Kategorie Nährstoffhaushalt und Emissionen mit den bereits erwähnten Toleranzbereichen und Boniturnoten zusammengefasst. Der Stickstoff-Saldo, der eine erste allgemeine Indikation einer betriebsbedingten Umweltwirkung indiziert, erhält die Boniturnote 4, da im Betrieb ein Nährstoff-Überschuss produziert wird. Da es sich um den korrigierten $\mathrm{N}$ Saldo (flächenbezogen; vorherige Ausweisung der Emissionen unter Verwendung des Pauschalansatzes aus der DüngeVO

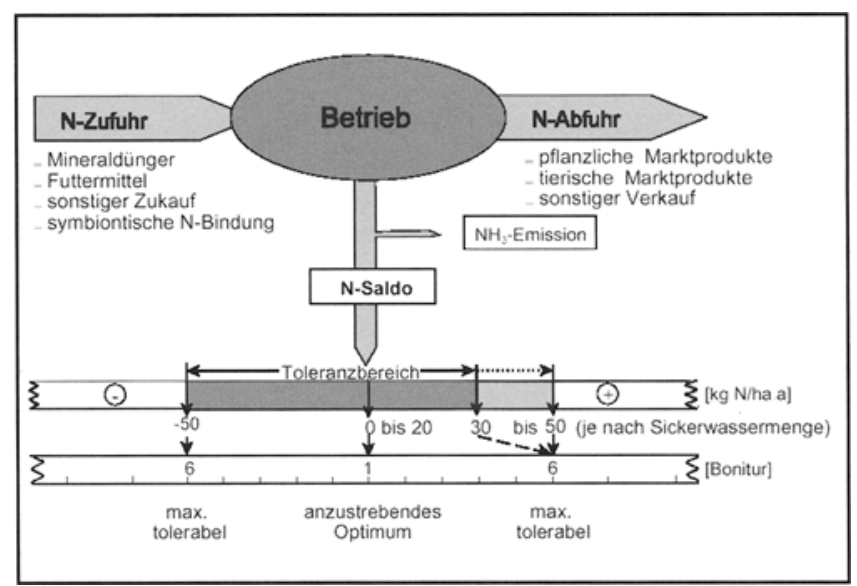

Abb. 2: KUL-Verfahrensweise zur Stickstoff-Bilanzierung (Quelle: VDLUFA 2000, S. 32)

Tab. 4: Übersicht aller Prüfkriterien und Betriebswerte in der Kategorie Nährstoffhaushalt und Emissionen für 1999

\begin{tabular}{|c|c|c|c|c|}
\hline Kriterium & Optimum & Toleranzbereich & Betriebswert Bakenhus & Boniturnote \\
\hline $\mathrm{N}$-Saldo [kg N/ha] & 20 & $-50 \ldots 50$ & 40,73 & 4 \\
\hline P-Saldo [kg P/ha] & 0 & $-15 \ldots 15$ & 1,59 & 1 \\
\hline $\mathrm{NH}_{3}$-Emissionen [kg NH$\left.{ }_{3}-\mathrm{N} / \mathrm{ha} \times \mathrm{a}\right]$ & 25 & $<50$ & 23,16 & 1 \\
\hline Gehaltsklasse P & $\mathrm{C}$ & $B \ldots D$ & C & 1 \\
\hline Gehaltsklasse K & C & B ... D & C & 1 \\
\hline Gehaltsklasse Mg & $\mathrm{C}$ & B ... D & $\mathrm{C}$ & 1 \\
\hline Boden $\mathrm{pH}$ - Stufe & $\mathrm{C}$ & $C \ldots D$ & $\mathrm{C}(\mathrm{pH} 5,3)$ & 1 \\
\hline
\end{tabular}


und der Betriebsdaten aus der Stallbilanz) handelt, soll hier eine mögliche, auf die Fläche gerichtete Belastung diagnostiziert wèrden. Unabhängig davon, ob für eine Flächenbilanz der Einzelschlag, die Fläche der einzelnen Fruchtfolgeglieder oder die gesamte Betriebsfläche der Bezugspunkt ist, werden durch sie die dem Boden zugeführten Nährstoffmengen (mineralische und organische Düngemittel, legume N-Fixierung, atmosphärische N-Einträge) und die entzogenen Nährstoffmengen (Ernteabfuhr) saldiert. Der Saldo entspricht den Nährstoffvorratsänderungen im Boden. Der N-Saldo einer Flächenbilanz eignet sich nach Bach (1987) zur Abschätzung der potenziellen Nitratbelastung des Grundwassers.

\subsection{Humusbilanz}

Eine der Grundvoraussetzungen für hohe Ackerbauerträge ist eine hohe natürliche Fruchtbarkeit der Böden, die zu einem erheblichen Teil vom Humusgehalt abhängt. Da Humus nur über relativ lange Zeiträume in größerem Umfang in unseren Ackerböden angereichert werden kann, der Abbau oder der Verlust durch Erosion jedoch unter ungünstigen Bedingungen wesentlich schneller verlaufen kann, ist eine Abschätzung des Humusverbrauches sowie der Humusnachlieferung von groBer Wichtigkeit für eine nachhaltige Aufrechterhaltung der Bodenfruchtbarkeit. Eine Methode der Humusbilanzierung ist die HE-Methode (Humuseinheit) nach Rauhe und Schönmeier (1966). Hier gilt: 1 Humuseinheit $(H E)=1$ ( $t$ ) Humus mit $50 \mathrm{~kg}$ Stickstoff und $580 \mathrm{~kg}$ Koblenstoff (Leithold et al. 1997 a, 1997 b). Nach Asmus und Hermann (1977) gilt: 1 Einheit reproduktionswirksame organische Substanz (ROS) $=1$ t organische Trockenmasse von Stalldung (Leithold et al. 1997a, S. 46). In der vorliegenden Arbeit werden Humuseinheiten (HE) berechnet und zur Verknüpfung mit KUL in ROS umgerechnet. Die Ergebnisse werden als Humusmehrungskoeffizienten (Versorgungsgrad des Bodens mit Humus in \%) angegeben. Die abschließende Boniturnote lässt sich auf dieser Basis berechnen und darstellen.

Die Datengrundlage wurde EDV-gestützt aufbereitet. Anschließend erfolgte eine Differenzierung in eine betriebs- und schlagbezogene Bilanzierung. Diese Bilanz erstreckt sich über die Jahre 1997 bis 2001. Die betriebsbezogene Humusbilanz für die Jahre 1997 bis 2000 kann Abb. 3 entnommen werden. Der Biohof Bakenhus erreicht für den Bereich der Humusbilanzierung eine auf das Jahr 2000 bezogene Boniturnote von 5: der Betriebswert für dieses Jahr weist einen Versorgungsgrad des Bodens mit Humus von 140,96\% auf und liegt somit am unteren Ende des Toleranzbereiches von 80 bis $150 \%$.

Die Humusbilanz unterscheidet sich von dem KUL-Ansatz auch dadurch, dass sie nicht rein betriebsbezogen, sondern auch schlagbezogen ist. Ferner beträgt der Bilanzzeitraum nicht ein Jahr, sondern umfasst eine Zeitspanne von 4 Jahren (19972000). Betrachtet man die betriebsbezogenen Bilanzen für die Jahre 1997 bis 2000 (Abb. 3), so werden fast ausnahmslos gute bis sehr gute Humusmehrungskoeffizienten erzielt. Besonders fällt nur das Bilanzjahr 1999 auf, welches einen sehr hohen Wert $(\sim 750 \%)$ erreicht. Dieser Sachverhalt veranlasste die Teilnehmer des Studienprojektes dazu, von der KULVorgabe (Berechnung des jährlichen Humussaldos) abzuweichen. Es sollte in Erwägung gezogen werden, von der Einjahresbetrachtung einer Humusbilanz abzusehen, da die $\mathrm{Hu}^{-}$

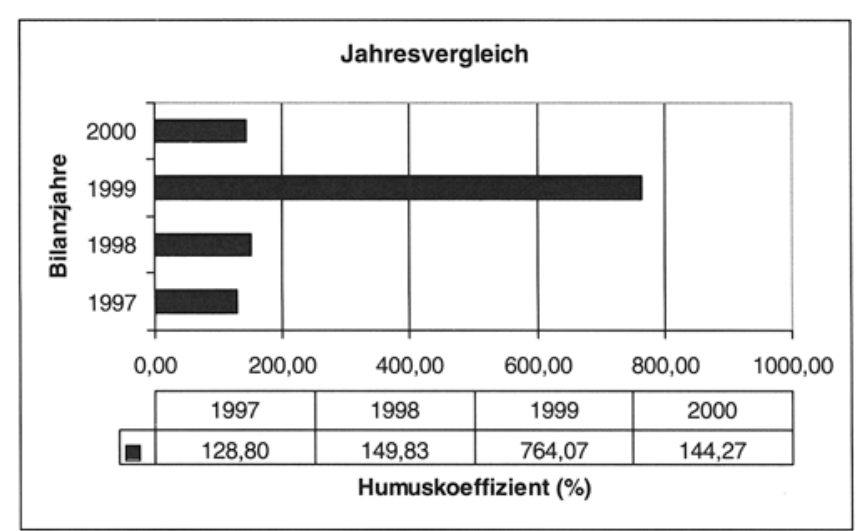

Abb. 3: Die betriebsbezogene Humusbilanz für die Jahre 1997 bis 2000 (eigene Darstellung)

musnachbildung wesentlich langsamer voranschreitet als die Humuszehrung. Betrachtet man das Anbaujahr 1999 alleine, so wird nicht deutlich, dass sich die Werte über einen mehrjährigen Zeitraum meist ausgleichen. Es werden Schläge z.T. in einem mehrjährigen Zeitraum auf eine humuszehrende Anbaufrucht vorbereitet. Das Jahr 2001 wurde zwar noch nicht in die Bilanz mit aufgenommen, die bereits vorliegenden Werte weisen aber darauf hin, dass es sich um ein sehr humuszehrendes Anbaujahr handeln wird, welches einen Ausgleich zu dem stark humusmehrenden Jahr 1999 darstellt.

\subsection{Tierhaltung}

Der Optimierungsvorschlag für KUL von Geier und Köpke (2000) sieht die Beurteilung der Tiergerechtheit vor. Als geeignete Methode zur einzelbetrieblichen Bewertung der Tiergerechtheit wird der Tiergerechtheitsindex TGI 200 nach Sundrum et al. (1994) vorgeschlagen (Geier und Köpke 2000, S. 86). In dem Studienprojekt wurde die Haltung der Schweine (Vor- und Endmast) und Rinder (differenziert nach Kälbern und erwachsenen Tieren) auf dem Biohof empirisch nach Sundrum et al. (1994) erfasst und bewertet. Die Bewertung erfolgt durch Einflussbereiche, welche essentielle Funktionskreise des Verhaltens darstellen (Sundrum et al. 1994, S. 22). Im einzelnen sind dies: Bewegungsverhalten, Nahrungsaufnahmeverhalten, Sozialverhalten, Ruheverhalten, Komfortverhalten, Ausscheidungsverhalten (nur Schweinehaltung), Hygiene und Betreuung.

Für jeden der Einflussbereiche existieren nach Tierarten differenzierte Bewertungstabellen. In diese Tabellen werden, je nach Ausprägung der einzelnen Merkmale, Punktzahlen eingetragen, die sich einem Bewertungsschlüssel entnehmen lassen. Die Summe aller erreichten Punkte stellt den TGI für die untersuchte Tierart dar. Das Optimum beträgt 200 Punkte; die tatsächlich erreichbare Punktzahl ist abhängig von der gegenwärtig praktizierten Haltungsform. Die Haltungsform der Rinder (Laufstallhaltung mit Auslauf und Weide) auf dem Biohof Bakenhus kann maximal mit einem TGI von 200 Punkten bewertet werden, die der Schweine (Mehrbereichssystem mit Auslauf) hingegen maximal mit einem TGI von 187 Punkten (Sundrum et al. 1994, S. 113, 174). Abb. 4 zeigt die Ergebnisse der empirischen Untersuchung oben genannter Kriterien auf dem Biohof Bakenhus. 


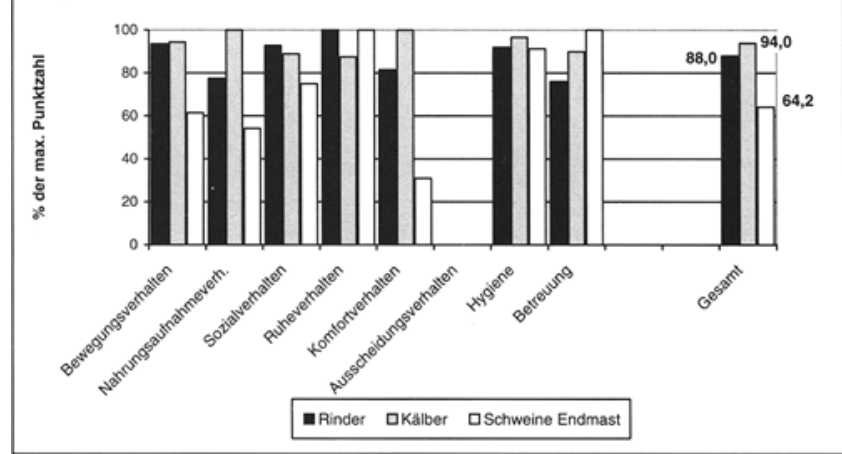

Abb. 4: Untersuchungsergebnisse der Tierhaltung auf dem Biohof Bakenhus (eigene Darstellung)

Der Bereich Ausscheidungsverhalten, welcher in Abb. 4 nicht mit Säulen versehen ist, bedarf der Erklärung: Für Rinder und Kälber ist dieser Einflussbereich nicht Gegenstand der Beurteilung, bei den Schweinen wurde er mit 0 bewertet und wird deshalb im Diagramm nicht dargestellt. Die Rinderund Kälberhaltung ist mit $88 \%$ bzw. $94 \%$ der erreichbaren Punktzahl als sehr zufrieden stellend einzustufen. Die niedrigere Bewertung der Schweinehaltung $(64,2 \%)$ resultiert v. a. aus einer mangelnden Strukturierung der Funktionsbereiche (Fressbereich, Wühlbereich, Kotbereich, Suhle, Auslauf,
Weide). Die Übertragung der Ergebnisse des TGI-200 in die von KUL geforderten Boniturnoten erfolgte unter Annahme eines Toleranzbereiches von $100 \%$ bis $50 \%$ der maximal erreichbaren Punktzahl. Die Boniturnoten 1 bis 6 wurden in gleichen Intervallen auf diesen Toleranzbereich verteilt. Dies ergab eine Boniturnote von 2 für die Rinderhaltung, 1 für die Kälberhaltung und 5 für die Schweinehaltung.

\subsection{Zusammenfassung der Ergebnisse des Wirkungsaudits}

Tabelle 5 liefert einen zusammenfassenden Soll-Ist-Vergleich und stellt die Boniturnoten für die einzelnen Umweltwirkungsbereiche.

\section{Umweltprogramm}

Das Umweltprogramm für den Biohof Bakenhus beschreibt auf Basis der ersten, bereichsübergreifenden Umweltprüfung die Maßnahmen, die getätigt werden sollten, um das Ziel einer kontinuierlichen Verbesserung des Umweltschutzes zu erreichen. Grundlage für die im folgenden dargestellten Handlungsempfehlungen bilden die im Wirkungsaudit für die jeweiligen Umweltwirkungsbereiche vergebenen Boniturnoten, die verbesserungswürdige Bereiche signalisieren und eine

Tab. 5: Zusammenfassender Soll-Ist-Vergleich mit den Boniturnoten für die einzelnen Umweltwirkungsbereiche (eigene Darstellung)

\begin{tabular}{|c|c|c|c|c|c|c|c|c|c|c|c|c|c|}
\hline $\begin{array}{c}\text { Umweltwirkungsb } \\
\text { ereich }\end{array}$ & Kriterium & Dimension & Optimum & Toleranzbereich & Betriebswert & 12 & 3 & 4 & 5 & \begin{tabular}{l|l}
6 & 7
\end{tabular} & 8 & 9 & 10 \\
\hline \multirow{8}{*}{ Nährstoffhaushalt } & N-Saldo (Fläche) & $\mathrm{kg} \mathrm{N} / \mathrm{ha}$ & 20 & $-50 \ldots 50$ & 40,73 & & & & & & & & \\
\hline & P-Saldo (Fläche) & $\mathrm{Kg} \mathrm{N} / \mathrm{ha}$ & 0 & $-15 \ldots 15$ & 1,59 & & & & & & & & \\
\hline & $\mathrm{NH}_{3}$-Emission (Tiere) & $\mathrm{kg} \mathrm{N} /$ ha LF & 25 & $<50$ & 23,16 & & & & & & & & \\
\hline & Gehaltsklasse P & Ohne & C & $B \ldots D$ & c & & & & & & & & \\
\hline & Gehaltsklasse K & Ohne & C & $B \ldots D$ & c & & & & & & & & \\
\hline & Gehaltsklasse Mg & Ohne & C & $B \ldots D$ & c & & & & & & & & \\
\hline & Boden-pH-Stufe & $A-E$ & C & $C \ldots D$ & 5,3 & & & & & & & & \\
\hline & Humussaldo & Versorgungsgrad (\%) & 100 & $80-150 \%$ & 140,96 & & & & & & & & \\
\hline \multirow{3}{*}{ Bodenschutz } & Erosionsdisposition & t/ ha/a & $<0,0942$ & $0,0942-2$ & 0,4094 & & & & & & & & \\
\hline & Verdichtungsgefährdung & $\mathrm{P}_{\mathrm{T}} / \mathrm{P}_{\mathrm{B}}$ & $<1$ & $1-1,25$ & o.A. & & & & & & & & \\
\hline & Median Feldgröße & $\mathrm{Ha}$ & $<10$ & $10-40$ & $<3,345$ & & & & & & & & \\
\hline \multirow{7}{*}{$\begin{array}{l}\text { Landschafts- und } \\
\text { Artenvielfalt }\end{array}$} & $\begin{array}{c}\text { Biodiversität (FRIEBEN } \\
\text { 1998) } \\
\end{array}$ & Punkte & 33 & & 17 & & & & & & & & \\
\hline & Verhältnis AF / GF & Punkte & 5 & & 2 & & & & & & & & \\
\hline & Düngungsniveau & Punkte & 5 & & 5 & & & & & & & & \\
\hline & Fruchtfolge & Punkte & 8 & & 4 & & & & & & & & \\
\hline & NBI & Punkte & 5 & & 3 & & & & & & & & \\
\hline & $\begin{array}{c}\text { Dichte mehrreihiger } \\
\text { Gehölze }\end{array}$ & Punkte & 5 & & 0 & & & & & & & & \\
\hline & $\begin{array}{c}\text { Präsenz von } \\
\text { Sonderbiotopen }\end{array}$ & Punkte & 5 & & 3 & & & & & & & & \\
\hline \multirow{4}{*}{ Tierhaltung } & TGI nach SUNDRUM & & & & & & & & & & & & \\
\hline & Rinder & Punkte & 200 & $200-100$ & 176 & & & & & & & & \\
\hline & Kälber & Punkte & 200 & $200-100$ & 187 & & & & & & & & \\
\hline & Schweine & Punkte & 200 & $200-100$ & 118,25 & & & & & & & & \\
\hline
\end{tabular}


grobe Abschätzung des Ausmaßes der durchzuführenden Optimierungen anzeigen.

Aus den Umweltwirkungen des Biohof Bakenhus wird die ökologische Wirtschaftsweise ersichtlich. Obwohl alle der Umweltprüfung unterzogenen Bereiche einer umweltverträglichen landwirtschaftlichen Nutzung zuzuordnen sind, lassen sich dennoch einige optimierungsfähige Schwachstellen aufzeigen. Um den Erhalt der natürlichen Lebensgrundlagen und eine naturgemäße Bewirtschaftung weiterhin zu gewährleisten, sollten folgende Handlungsempfehlungen berücksichtigt werden:

- Bodenschutz

- Verzicht auf Ackerfrüchte mit hohen relativen Bodenabtragsraten (insbes. Mais)

- Anpflanzung von Hecken und Wällen unter Berücksichtigung von Windrichtungen und Hangneigung

- Verkleinerung von größeren Schlägen durch Hecken und Wälle

- Biodiversität

- Beachtung der Flächenverteilung von Kulturen bei einer Fruchtfolge

- Eventuell Verkleinerung von größeren Schlägen durch Hecken und Wälle in Anlehnung an die in der Preußischen Uraufnahme abgebildeten Verhältnisse, auch im Hinblick auf die Verringerung von Erosion

- Ausweitung der Obstwiesen

- Tierhaltung

- Verringerung der Trogbodenhöhe (Rinder)

- Aufteilung des Schweinestalles und -auslaufes in mehrere Bereiche

- Fütterung der Schweine 'ad libitum' oder dreimal täglich

- Schaffung von Einrichtungen für Komfort / Erkundungsverhalten der Schweine

- Ergänzen der Einstreu für Schweine durch weitere Bestandteile

- Gruppenauslaufhaltung bzw. ganzjährige Haltung auf der Weide mit Witterungsschutz für die Pferde

- Vergrößerung der Pferdeboxen

Der Umweltwirkungsbereich Bodenschutz ist ähnlich wie der des Nährstoffhaushalts oder der Humusbilanz durchgehend positiv bewertet worden, weshalb die ausgesprochenen Empfehlungen als nachrangig einzustufen sind und die Optimierung der übrigen Bereiche vorrangig behandelt werden sollte. Zur Biodiversität sind vorwiegend Handlungsempfehlungen im Hinblick auf die Fruchtfolge sowie die Dichte mehrreihiger Gehölze gegeben. Bei der Tierhaltung besteht lediglich bei den Schweinen Handlungsbedarf, welcher auch direkte ökonomische Folgen nach sich ziehen dürfte.

\section{Schlussbetrachtung}

Die Implementierung eines Umweltmanagementsystems für die landwirtschaftliche Produktion, das sich an standardisierten Verfahren orientiert, ist nach den Ergebnissen des Studienprojektes bei ausreichender Datengrundlage realisierbar. Es ist jedoch darauf hinzuweisen, dass der Verordnungstext der EG-Öko-Audit-Verordnung keinerlei Verfahrensweisen oder Prüfkriterien für die Landwirtschaft vorgibt. Somit ist es erforderlich, ein wissenschaftlich abgesichertes wie handhabbares Modell zu etablieren. Adäquate Instrumente (REPRO, KUL) werden derzeit erprobt. Zukünftig wird es zu einer Harmonisierung dieser Verfahren kommen müssen, um eine normative Grundlage für das Umweltmanagement im Bereich der Agrarproduktion schaffen zu können. Die Auswahl der Agrar-Umweltindikatoren sollte vor dem Hintergrund der Datenverfügbarkeit und der Berechnung bzw. Bilanzierung erfolgen.

Der Bodenschutz als wesentlicher Prüfbereich umweltverträglicher Produktion ist, wie das Wirkungsaudit im Studienprojekt gezeigt hat, nur unter erheblichem Datenerhebungs- und Berechnungsaufwand mit aussagekräftigen Ergebnissen darstellbar. Die einzelbetriebliche Ebene kann diesen Aufwand aus arbeitswirtschaftlichen und finanziellen Gründen nur bedingt leisten. Hier wird eine konsequente Kooperation von Forschung und Praxis sowie der öffentlichen Stellen erforderlich.

$\mathrm{Da}$ es sich bei dem Untersuchungsbetrieb um einen nach NATURLAND-Richtlinien wirtschaftenden Betrieb handelt, ist eine Übertragung der Erkenntnisse auf konventionelle Betriebe nur ansatzweise möglich. Mit der ökologischen Ausrichtung unterziehen sich die Betriebe bereits einer freiwilligen, regelmäßigen Prüfung durch entsprechende Kontrollstellen. Für die anderen Betriebe gelten die Vorgaben aus den gesetzlichen und untergesetzlichen Regelwerken (Düngemittelgesetz, DüngeVO, Tierschutzgesetz etc.). Diese sind aufsichtsbehördlich prüfbar und ebenfalls einzuhaiten. Das Umweltmanagement fördert in diesem Falle die Außenwirkung und Transparenz der Normen.

Den Aspekten der Außenwirkung und Transparenz sollte größte Beachtung geschenkt werden, um das oft negative Image der konventionellen Betriebe in der Öffentlichkeit zu durchbrechen. Diese Chance muss die Agrarwirtschaft gerade vor dem Hintergrund der politischen Diskussion einer Agrarwende zur Ökologisierung der Landwirtschaft nutzen. Neben den aktuellen Entwicklungen im Bereich der Produktzertifizierung (Öko-Siegel) ist die betriebliche Produktion ebenfalls nach ökologischen Gesichtspunkten zu beurteilen. Das EG-Öko-Audit ist als strukturelles Management-Verfahren für die Betriebsprüfung geeignet.

\section{Literatur}

Asmus F, Hermann V (1977): Reproduktion der organischen Substanz des Bodens. Berlin

Auerswald K, Schwertmann U (1988): Modelle zur Erosionsvorhersage als Entscheidungsgrundlage des Bodenschutzes. In: Rosenkranz et al. (Hrsg), BoS 1. Lfg XI / 88, Zf. 4085

Bach M (1987): Die potentielle Nitrat-Belastung des Sickerwassers durch die Landwirtschaft in der Bundesrepublik Deutschland. Göttingen (Göttinger Bodenkundliche Berichte 93)

Biohof Bakenhus (2000): Betriebsspiegel Biohof Bakenhus Landwirtschaft. Großenkneten

BGR (Bundesanstalt für Geowissenschaften und Rohstoffe) (Hrsg.) (1982): Bodenkundliche Kartieranleitung. Hannover, 3. verbesserte und erweiterte Auflage

BMELF (Bundesministerium für Ernährung, Landwirtschaft und Forsten) (Hrsg) (1991): Berichte über Landwirtschaft - Zeitschrift für Agrarpolitik und Landwirtschaft. Hamburg / Berlin, Sonderheft Nr 205, Reihe: Bodennutzung und Bodenfruchtbarkeit, Bd 3: Bodenerosion

BMELF (Hrsg) (1996): Verordnung über die Grundsätze der guten fachlichen Praxis beim Düngen (Düngeverordnung) vom 26. Januar 1996. BGBl Teil 1. Vom 06. Februar 1996, S. 118, geändert durch Artikel 2 der Verordnung vom 16. Juli 1997, BGBl Teil I, S 1836 
BMELF (Hrsg.) (2000a): Berichte über Landwirtschaft. Zeitschrift für Agrarpolitik und Landwirtschaft. Münster-Hiltrup, Bd 78 (1), März 2000

BMELF (Hrsg.) (2000b): Berichte über die Landwirtschaft. Zeitschrift für Agrarpolitik und Landwirtschaft. Münster-Hiltrup, Bd 78 (3), September 2000

Diepenbrock W, Kaltschmitt M, Nieberg H, Reinhardt G (Hrsg) (1997): Umweltverträgliche Pflanzenproduktion. Indikatoren, Bilanzierungsansätze und ihre Einbindung in Ökobilanzen. Osnabrück

Doluschitz R, Welk H, Zeddies J (1992): Stickstoffbilanzen landwirtschaftlicher Betriebe. Ein Einstieg in die ökologische Buchführung? Berichte über Landwirtschaft, Bd 70

Dubsky G, Hülsbergen KJ, Diepenbrock W (1997): Vortrag auf dem 7. Treffen der Arbeitsgruppe Werkzeuge für Modellbildung und Simulation in Umweltanwendungen im Fachausschuss 4.6. Informatik im Umweltschutz der Gesellschaft für Informatik e.V., Oldenburg, 5./6. Juni 1997. In: Keller H, Grützner R, Sonnenschein M (1999)

Eckert H, Breitschuh G (1997): Kritische Umweltbelastung Landwirtschaft (KUL): Ein Verfahren zur Erfassung und Bewertung landwirtschaftlicher Umweltwirkungen. In: Diepenbrock et al. (Hrsg), S 185-195

Eckert H, Breitschuh G, Sauerbeck DR (1999): Kriterien umweltverträglicher Landbewirtschaftung (KUL). Ein Verfahren zur ökologischen Bewertung von Landwirtschaftsbetrieben. Agribiological Research, Heft 52 (1), S 57-76

Frieben B (1998): Verfahren zur Bestandsaufnahme und Bewertung von Betrieben des Organischen Landbaus im Hinblick auf Biotop- und Artenschutz und die Stabilisierung des Agrarökosystems. Berlin (Schriftenreihe Institut für Organischen Landbau)

FOSVWE (2000): Ergebnisse der Beprobung von Testflächen auf dem Biohof Bakenhus. Vechta

Gebauer J, Bäurle AS (2000): Betriebliche Umweltinformationstechniken für die Landwirtschaft. In: BMELF (2000b)

Geier U, Köpke U (2000): Analyse und Optimierung des betrieblichen Umweltbewertungsverfahrens, Kriterien umweltverträglicher Landbewirtschaftung' (KUL). In: BMELF (2000 a), S 70-91

Hjulström F (1935): Studies on the morphological activities of rivers as illustrated by the river Fyris. Bulletin of the Geological Institute of Upsala 25, S 221-527

Hülsbergen K-J, Biermann S (1994): Modelle zur Bilanzierung von Nährstoffflüssen in Agroöokosystemen. Mitteilungen der Gesellschaft für Pflanzenbauwissenschaften, Bd 7, S 207-210

Keller H, Grützner R, Sonnenschein M (Hrsg) (1999): 7. Treffen des Arbeitskreises Werkzeuge der Simulation und Modellbildung in Umweltanwendungen der Gesellschaft für Informatik. Karlsruhe (Forschungszentrum Karlsruhe FZKA, Wissenschaftliche Berichte)

LK OL (Landkreis Oldenburg) (Hrsg) (1995): Landschaftsrahmenplan 1995. Wildeshausen

LWK WE (Landwirtschaftskammer Weser-Ems (1999): Düngeplanung für das Jahr 1999. Oldenburg

Leithold G, Hülsbergen KJ, Michel D, Schönmeier H (1997a): Humusbilanzierung. Methoden und Anwendung als Agrar-Umweltindikator. In: Diepenbrock et al. (Hrsg), S 43-54

Leithold G, Hülsbergen KJ, Michel D, Schönmeier H (1997b): Humusbilanzierung - Methoden und Anwendungen als Agrar-Umweltindikator. Schriftenreihe der Sächsischen Landesanstalt für Landwirtschaft, Heft 3, 2. Jg, S 19-28

Münchhausen H, Nieberg H (1997): Agrar-Umweltindikatoren: Grundlagen, Verwendungsmöglichkeiten und Ergebnisse einer Expertenbefragung. In: Diepenbrock et al. (Hrsg), S 13-28

Paul R (1999): Zur Verdichtungsgefährdung im Rahmen des Bodengefügeschutzes auf großen Flächen. In: TMLNU (Hrsg), S 54-59

Rauhe K, Schönmeier H (1966): Über die Bedeutung des Humusersatzes beim Übergang zu industriemäßigen Produktionsmethoden. Wissenschaftliche Zeitschrift der Karl-Marx-Universität-Leipzig, mathematisch-naturwissenschaftliche Reihe 15, Heft 1, S 1-5

Rosenkranz D, Einsele G, Harress HM, Bachmann G (Hrsg) (1988): Bodenschutz. Ergänzbares Handbuch der Maßnahmen und Empfehlungen für Schutz, Pflege und Sanierung von Böden, Landschaft und Grundwasser. Berlin, 3 Bde

Schneider B (1999): Pilotprojekt zur Anwendung der EG-Umwelt-Audit-Verordnung auf landwirtschaftliche Betriebe: Schweinemastbetriebe. Berlin, UBA-Text 94/99.
Schröder W (1996): Einsatz von Biosphärenreservaten für Integrative Umweltbeobachtung und -bewertung sowie Naturschutz. Beiträge der Akademie für Natur- und Umweltschutz Baden-Württemberg Bd 23, S 143-167

Schröder W (1998a): Ökologie und Umweltrecht als Herausforderung natur- und sozialwissenschaftlicher Forschung und Lehre. In: Daschkeit A, Schröder W (Hrsg): Perpektiven integrativer Umweltforschung. Berlin u.a. (Umweltnatur- und Umweltsozialwissenschaften UNS Bd 1), S 329-357

Schröder W (1998b): Nachhaltige Bodennutzung und Welternährungslage. In: Verhandlungen des 51. Deutschen Geographentages: Stuttgart, S 105-124

Schröder W (1999): Empirisch-ökologische und normativ-juristische Argumentation. In: Gerold G, Schneider-Sliwa R, Schaub D (Hrsg): Angewandte Landschaftsökologie. Grundlagen und Methoden. Berlin (ua), S 451-475

Schröder W, Daschkeit A (1994): Ökosystemare Umweltbewertung. Thesen zum Anforderungsprofil zukünftiger Umweltforschung. UWSF - Umweltwissenschaften und Schadstoff-Forschung. Zeitschrift für Umweltchemie und Ökotoxikologie 6 (3) S 139-144

Schröder W, Daschkeit A (2002): Ökologische, ökonomische und rechtliche Umweltbewertung. Forschungs- und Lehrgegenstand eines Graduiertenkollegs. In: UWSF - Umweltwissenschaften und Schadstoff-Forschung. Zeitschrift für Umweltchemie und Ökotoxikologie 14 (3) $178-186$

Schwertmann U, Vogl W, Kainz M (1987): Bodenerosion durch Wasser. Vorhersage des Abtrags und Bewertung von Gegenmaßnahmen. Stuttgart

Sundrum A, Andersson R, Postler G (1994): Tiergerechtheitsindex 200/ 1994. Ein Leitfaden zur Beurteilung von Haltungssystemen. Bonn

VAFB (Verband für Agrarforschung und -bildung e.V. Jena) (1999): Erfahrungen und Schlussfolgerungen des Öko-Audits in Thüringer Landwirtschaftsbetrieben. Jena

TMLNU (Hrsg) (1999): Einfluss der Großflächen-Landwirtschaft auf den Boden. Jena (Kolloquium Tierschutzgesetz in der Fassung vom 25.5.1998)

VLB (Verband der Landwirtschaftsberater in Bayern e.V.) (Hrsg) (1987): Pflanzliche Erzeugung: Grundlagen der Pflanzenproduktion, umweltbewusster Landbau, Produktionstechnik der Kulturpflanzen, Futterkonservierung, Dauergrünland. München (Die Landwirtschaft, Bd 1)

VDLUFA (Verband Deutscher Landwirtschaftlicher Untersuchungs- und Forschungsanstalten (Hrsg) (1998): Kriterien umweltverträglicher Landbewirtschaftung. VDLUFA-Standpunkt

VDLUFA (Verband Deutscher Landwirtschaftlicher Untersuchungs- und Forschungsanstalten (Hrsg) (2000): Kongressband 2000 StuttgartHohenheim, Generalthema Nachhaltige Landwirtschaft, Teil 1, Vorträge zur Plenartagung. 112. VDLUFA-Kongress vom 18.-22. September 2000. VDLUFA Schriftenreihe 55/2000. Darmstadt

White ID, Mottershead DN, Harrison SJ (1984): Environmental systems. London

Wischmeier WH, Smith DD (1978): Predicting rainfall erosion losses. Washington (USDA-Agricultural Handbook 537)

Zellmann T, Bäuerle A, Doluschitz R, Jahnke D, Marell D (1999a): Braucht die Landwirtschaft Umweltmanagementsysteme? UWSF Umweltwissenschaften und Schadstoff-Forschung 11 (1) S 49-54 (Teil I der Beitragsserie: Umweltmanagement im landwirtschaftlichen Betrieb)

Zellmann T, Bäuerle A, Doluschitz R, Jahnke D, Marell D (1999b): Lösungsansätze für den landwirtschaftlichen Betrieb bei der Implementierung eines Umweltmanagementsystems? UWSF - Umweltwissenschaften und Schadstoff-Forschung 11 (3) S 181-186 (Teil II der Beitragsserie: Umweltmanagement im landwirtschaftlichen Betrieb)

Zitierte Internet-Seite: http://mapserver.ilpoe.uni-stuttgart.de:81/krabag/ abag.html

Eingegangen: 9. August 2002 Akzeptiert: 10. September 2002 OnlineFirst: 13. September 2002 